\title{
Commuting Quantum Circuits with Few Outputs are Unlikely to be Classically Simulatable
}

\author{
Yasuhiro Takahashi \\ NTT Communication Science Laboratories, \\ NTT Corporation \\ takahashi.yasuhiro@lab.ntt.co.jp \\ Takeshi Yamazaki \\ Mathematical Institute, Tohoku University \\ yamazaki@math.tohoku.ac.jp
}

\author{
Seiichiro Tani \\ NTT Communication Science Laboratories, \\ NTT Corporation \\ tani.seiichiro@lab.ntt.co.jp \\ Kazuyuki Tanaka \\ Mathematical Institute, Tohoku University \\ tanaka@math.tohoku.ac.jp
}

\begin{abstract}
We study the classical simulatability of commuting quantum circuits with $n$ input qubits and $O(\log n)$ output qubits, where a quantum circuit is classically simulatable if its output probability distribution can be sampled up to an exponentially small additive error in classical polynomial time. First, we show that there exists a commuting quantum circuit that is not classically simulatable unless the polynomial hierarchy collapses to the third level. This is the first formal evidence that a commuting quantum circuit is not classically simulatable even when the number of output qubits is exponentially small. Then, we consider a generalized version of the circuit and clarify the condition under which it is classically simulatable. Lastly, we apply the argument for the above evidence to Clifford circuits in a similar setting and provide evidence that such a circuit augmented by a depth-1 non-Clifford layer is not classically simulatable. These results reveal subtle differences between quantum and classical computation.
\end{abstract}

\section{Introduction and Summary of Results}

One of the most important challenges in quantum information processing is to understand the difference between quantum and classical computation. An approach to meeting this challenge is to study the classical simulatability of quantum computation. Previous studies have shown that restricted models of quantum computation, such as commuting quantum circuits, are useful for this purpose [20, 5, 17, 16, 2, 3, 12, 8, 19, 11]. Because of the simplicity of such restricted models, they are also useful for identifying the source of the computational power of quantum computers. It is therefore of great interest to study their classical simulatability.

In this paper, we study the classical simulatability of commuting quantum circuits with $n$ input qubits and $O(\operatorname{poly}(n))$ ancillary qubits initialized to $|0\rangle$, where a commuting quantum circuit is a quantum circuit consisting of pairwise commuting gates, each of which acts on a constant number of qubits. When all commuting gates in a commuting quantum circuit act on at most $c$ qubits for some constant $c \geq 2$, the circuit is said to be $c$-local. For considering the classical simulatability, we adopt strong and weak simulations. The strong simulation of a quantum circuit is to compute its output probability up to an exponentially small additive error in classical polynomial time and the weak one is to sample its output probability distribution similarly. Any strongly simulatable quantum circuit is weakly simulatable. Our main focus is on the hardness of classically simulating quantum circuits and thus we mainly deal with the weak simulatability, which yields a stronger result than that the strong simulatability yields. Previous hardness results on the weak simulatability are 
usually obtained with respect to multiplicative error [20, 3, 8], but such an error seems to be too strong an assumption as discussed in [2]. Our results are obtained with respect to additive error.

In 2011, Bremner et al. showed that there exists a 2-local IQP circuit with $O(\operatorname{poly}(n))$ output qubits such that it is not weakly simulatable (under a plausible assumption) [3], where an IQP circuit is a quantum circuit consisting of pairwise commuting gates that are diagonal in the $X$ basis $\{(|0\rangle \pm|1\rangle) / \sqrt{2}\}$. Roughly speaking, this result means that when the number of output qubits is large, even a simple commuting quantum circuit is powerful. On the other hand, in 2013, Ni et al. showed that any 2-local commuting quantum circuit with $O(\log n)$ output qubits is strongly simulatable and that there exists a 3-local commuting quantum circuit with only one output qubit such that it is not strongly simulatable (under a plausible assumption) [12. Thus, when the number of output qubits is $O(\log n)$, the classical simulatability of commuting quantum circuits depends on the number of qubits affected by each commuting gate. A natural question is whether there exists a commuting quantum circuit with $O(\log n)$ output qubits such that it is not weakly simulatable.

There are two previous results related to this question. The first one is that any (constant-local) IQP circuit with $O(\log n)$ output qubits is weakly simulatable [3]. Thus, if we want to answer the above question affirmatively, we need to consider commuting quantum circuits other than IQP circuits. The second one is that, if any commuting quantum circuit with only one output qubit is weakly simulatable, there exists a polynomial-time classical algorithm for the problem of estimating the matrix element $|\langle 0|U| 0\rangle|$ (up to a polynomially small additive error) for any unitary matrix $U$ that is implemented by a constant-depth quantum circuit [12. This suggests an affirmative answer to the above question since the matrix element estimation problem seems to be hard for a classical computer. However, the hardness has not been formally understood yet.

We provide the first formal evidence for answering the above question affirmatively:

Theorem 1. There exists a 5-local commuting quantum circuit with $O(\log n)$ output qubits such that it is not weakly simulatable unless the polynomial hierarchy $\mathrm{PH}$ collapses to the third level.

It is widely believed that PH does not collapse to any level [15]. Thus, the circuit in Theorem 1 is the desired evidence. To construct the circuit, we first show the existence of a depth-3 quantum circuit $A_{n}$ that is not weakly simulatable with respect to additive error (under a plausible assumption), where it has $n$ input qubits, $O(\operatorname{poly}(n))$ ancillary qubits, and $O(\operatorname{poly}(n))$ output qubits. This is shown by our new analysis of the weak simulatability (with respect to additive error) of a depth3 quantum circuit that is not weakly simulatable with respect to multiplicative error (under a plausible assumption) [3, 5. Our idea for constructing the circuit in Theorem 1 is to combine $A_{n}$ with the OR reduction circuit [7, which reduces the computation of the OR function on $k$ bits to that on $O(\log k)$ bits. The resulting circuit has $O(\log n)$ output qubits and is not weakly simulatable (under a plausible assumption). It is of course not a commuting quantum circuit, but an important observation is that the OR reduction circuit can be transformed into a 2-local commuting quantum circuit. We consider a quantum circuit consisting gates of the form $A_{n}^{\dagger} g A_{n}$ for any commuting gate $g$ in the commuting OR reduction circuit and analyze it rigorously, which implies Theorem 1 .

Then, in order to generalize the above-mentioned result that any IQP circuit with $O(\log n)$ output qubits is weakly simulatable [3], we consider the weak simulatability of a generalized version of the circuit in Theorem 1. We assume that we are given two quantum circuits: $F_{n}$ is a quantum circuit with $n$ input qubits, $O(\operatorname{poly}(n))$ ancillary qubits, and $O(\operatorname{poly}(n))$ output qubits and $D$ is a quantum circuit on $O(\operatorname{poly}(n))$ qubits consisting of pairwise commuting gates that are diagonal in the $Z$-basis $\{|0\rangle,|1\rangle\}$. The generalized version is the circuit $\left(F_{n}^{\dagger} \otimes H^{\otimes l}\right) D\left(F_{n} \otimes H^{\otimes l}\right)$, where $l=O(\log n)$. The input qubits and output qubits of the circuit are the input qubits of $F_{n}$ and the ancillary qubits on which $H^{\otimes l}$ is applied, respectively. In particular, when $F_{n}=A_{n}$ and $D$ is a quantum circuit consisting of controlled phase-shift gates, the whole circuit becomes the circuit in Theorem 1. We show that the weak simulatability of $F_{n}$ implies that of the whole circuit: 
Theorem 2. If $F_{n}$ is weakly simulatable, then $\left(F_{n}^{\dagger} \otimes H^{\otimes l}\right) D\left(F_{n} \otimes H^{\otimes l}\right)$ with $l=O(\log n)$ output qubits is also weakly simulatable.

The above-mentioned result in [3] corresponds to the case when $F_{n}$ is a tensor product of $H$. Theorem 2 implies an interesting suggestion on how to improve Theorem 11. As described above, the 5-local commuting quantum circuit in Theorem 1 is constructed by choosing a depth-3 quantum circuit as $F_{n}$. A possible way to improve Theorem 1 , or more concretely, a possible way to construct a 3- or 4-local commuting quantum circuit that is not weakly simulatable would be to somehow choose a depth-2 quantum circuit as $F_{n}$. Theorem 2 implies that such a construction is impossible. This is because, since any depth-2 quantum circuit is weakly simulatable [20, 10], choosing a depth-2 quantum circuit as $F_{n}$ yields only a weakly simulatable quantum circuit.

We show Theorem 2 by simply generalizing the proof of the above-mentioned result in [3]. More precisely, we fix the states of the qubits other than the $O(\log n)$ output qubits on the basis of the assumption in Theorem 2 and then follow the change of the states of the output qubits. This yields a polynomial-time classical algorithm for weakly simulating $\left(F_{n}^{\dagger} \otimes H^{\otimes l}\right) D\left(F_{n} \otimes H^{\otimes l}\right)$.

Lastly, we apply the argument for proving Theorem 1 to Clifford circuits with $n$ input qubits, $O($ poly $(n))$ ancillary qubits in a product state, and $O(\log n)$ output qubits. A simple extension of the proof in [4, 8] implies that any Clifford circuit in the setting is strongly simulatable. We provide evidence that a slightly extended circuit is not weakly simulatable:

Theorem 3. There exists a Clifford circuit augmented by a depth-1 non-Clifford layer with $O(\operatorname{poly}(n))$ ancillary qubits in a particular product state and with $O(\log n)$ output qubits such that it is not weakly simulatable unless $\mathrm{PH}$ collapses to the third level.

Similar to Theorems 1 and 2, Theorem 3 contributes to understanding a subtle difference between quantum and classical computation. As in the proof of Theorem 1, using the result in [8], we show the existence of a Clifford circuit that is not weakly simulatable with respect to additive error (under a plausible assumption), where it has $n$ input qubits, $O(\operatorname{poly}(n))$ ancillary qubits in a particular product state, and $O(\operatorname{poly}(n))$ output qubits. Then, we combine the Clifford circuit with a constant-depth OR reduction circuit with unbounded fan-out gates [7]. The resulting circuit has $O(\log n)$ output qubits and is not weakly simulatable (under a plausible assumption). By decomposing the unbounded fan-out gates into CNOT gates, we transform the combination of the Clifford circuit and OR reduction circuit into a Clifford circuit augmented by a depth-1 non-Clifford layer, which implies Theorem 3. A similar argument with a constant-depth quantum circuit for the OR function with unbounded fan-out gates [18] implies that the number of output qubits can further be decreased to one at the cost of adding one more depth-1 non-Clifford layer.

\section{Preliminaries}

\subsection{Quantum Circuits}

We use the standard notation for quantum states and the standard diagrams for quantum circuits [13. The elementary gates in this paper are a Hadamard gate $H$, a phase-shift gate $R(\theta)$ with angle $\theta= \pm 2 \pi / 2^{k}$ for any $k \in \mathbb{N}$, and a controlled- $Z$ gate $\Lambda Z$, where

$$
H=\frac{1}{\sqrt{2}}\left(\begin{array}{cc}
1 & 1 \\
1 & -1
\end{array}\right), R(\theta)=\left(\begin{array}{cc}
1 & 0 \\
0 & e^{i \theta}
\end{array}\right), \Lambda Z=\left(\begin{array}{cccc}
1 & 0 & 0 & 0 \\
0 & 1 & 0 & 0 \\
0 & 0 & 1 & 0 \\
0 & 0 & 0 & -1
\end{array}\right)
$$

We denote $R(\pi), R(\pi / 2)$, and $H R(\pi) H$ as $Z, P$, and $X$, respectively, where $Z$ and $X$ (with $Y=i X Z$ and identity $I$ ) are called Pauli gates. We also denote $H \Lambda Z H$ as $\Lambda X$, which is a CNOT 
gate, where $H$ acts on the target qubit. A quantum circuit consists of the elementary gates. In particular, when a quantum circuit consists only of $H, P$, and $\Lambda Z$, it is called a Clifford circuit. A commuting quantum circuit is a quantum circuit consisting of pairwise commuting gates, where we do not require that each commuting gate be one of the elementary gates. In other words, when we think of a quantum circuit as a commuting quantum circuit, we are allowed to regard a group of elementary gates in the circuit as a single gate and we require that such gates, which are not necessarily elementary gates, be pairwise commuting.

The complexity measures of a quantum circuit are its size and depth. The size is the number of elementary gates in the circuit. To define the depth, we consider the circuit as a set of layers $1, \ldots, d$ consisting of one-qubit and two-qubit gates, where gates in the same layer act on pairwise disjoint sets of qubits and any gate in layer $j$ is applied before any gate in layer $j+1$. The depth of the circuit is the smallest possible value of $d[5]$. It seems to be natural to require that each gate in a layer be one of the elementary gates, but we do not require this for simplicity and we consider one-qubit and two-qubit gates determined from the context. In other words, when we count the depth, we are allowed to consider one-qubit and two-qubit gates generated by elementary gates in the circuit. Regardless of whether we adopt the requirement or not, the depth of the circuit we are interested in is a constant. A quantum circuit can use ancillary qubits initialized to $|0\rangle$. We do not require that the states of the ancillary qubits be reset to $|0\rangle$ at the end of the computation.

We deal with a uniform family of polynomial-size quantum circuits $\left\{C_{n}\right\}_{n \geq 1}$, where each $C_{n}$ is a quantum circuit with $n$ input qubits and $O(\operatorname{poly}(n))$ ancillary qubits, and can use phase-shift gates with angles $\theta= \pm 2 \pi / 2^{k}$ for any $k=O(\operatorname{poly}(n))$. Some of the input and ancillary qubits are called output qubits. At the end of the computation, $Z$-measurements, i.e., measurements in the $Z$-basis, are performed on the output qubits. The uniformity means that there exists a polynomial-time deterministic classical algorithm for computing the function $1^{n} \mapsto \overline{C_{n}}$, where $\overline{C_{n}}$ is the classical description of $C_{n}$. A symbol denoting a quantum circuit, such as $C_{n}$, also denotes its matrix representation in some fixed basis. Any quantum circuit in this paper is understood to be an element of a uniform family of polynomial-size quantum circuits and thus, for simplicity, we deal with a quantum circuit $C_{n}$ in place of a family $\left\{C_{n}\right\}_{n \geq 1}$. We require that each commuting gate in a commuting quantum circuit act on a constant number of qubits. When all commuting gates act on at most $c$ qubits for some constant $c \geq 2$, the circuit is said to be $c$-local [12].

\subsection{Classical Simulatability and Complexity Classes}

We deal with a uniform family of polynomial-size classical circuits to model a polynomial-time deterministic classical algorithm. Similarly, to model its probabilistic version, we deal with a uniform family of polynomial-size randomized classical circuits, each of which has a register initialized with random bits for each run of the computation [3]. As in the case of quantum circuits, for simplicity, we consider a classical circuit in place of a family of classical circuits.

Let $C_{n}$ be a polynomial-size quantum circuit with $n$ input qubits, $O(\operatorname{poly}(n))$ ancillary qubits, and $m$ output qubits. For any $x \in\{0,1\}^{n}$, there exists an output probability distribution $\left\{\left(y, \operatorname{Pr}\left[C_{n}(x)=y\right]\right)\right\}_{y \in\{0,1\}^{m}}$, where $\operatorname{Pr}\left[C_{n}(x)=y\right]$ is the probability of obtaining $y \in\{0,1\}^{m}$ by $Z$-measurements on the output qubits of $C_{n}$ with the input state $|x\rangle$. The classical simulatability of $C_{n}$ is defined as follows [20, 21, 3, 22, 12, 8, 19]:

Definition 1. $-C_{n}$ is strongly simulatable if the output probability $\operatorname{Pr}\left[C_{n}(x)=y\right]$ and its marginal output probability can be computed up to an exponentially small additive error in classical $O(\operatorname{poly}(n))$ time. More precisely, for any polynomial $p$, there exists a polynomial-size classical circuit $D_{n}$ such that, for any $x \in\{0,1\}^{n}$ and $y \in\{0,1\}^{m}$,

$$
\left|D_{n}(x, y)-\operatorname{Pr}\left[C_{n}(x)=y\right]\right| \leq \frac{1}{2^{p(n)}}
$$


and, when we choose arbitrary $m^{\prime}$ output qubits from the $m$ output qubits of $C_{n}$ for any $m^{\prime}<m$, the output probability $\operatorname{Pr}\left[C_{n}(x)=y^{\prime}\right]$ can be computed similarly for any $x \in\{0,1\}^{n}$ and $y^{\prime} \in\{0,1\}^{m^{\prime}}$.

- $C_{n}$ is weakly simulatable if the output probability distribution $\left\{\left(y, \operatorname{Pr}\left[C_{n}(x)=y\right]\right)\right\}_{y \in\{0,1\}^{m}}$ can be sampled up to an exponentially small additive error in classical $O(\operatorname{poly}(n))$ time. More precisely, for any polynomial $p$, there exists a polynomial-size randomized classical circuit $R_{n}$ such that, for any $x \in\{0,1\}^{n}$ and $y \in\{0,1\}^{m}$,

$$
\left|\operatorname{Pr}\left[R_{n}(x)=y\right]-\operatorname{Pr}\left[C_{n}(x)=y\right]\right| \leq \frac{1}{2^{p(n)}} .
$$

Any strongly simulatable quantum circuit is weakly simulatable [20, 3].

The following two complexity classes are important for our discussion [1, 3, 6]:

Definition 2. Let $L \subseteq\{0,1\}^{*}$.

- $L \in$ PostBQP if there exists a polynomial-size quantum circuit $C_{n}$ with $n$ input qubits, $O(\operatorname{poly}(n))$ ancillary qubits, one output qubit, and one particular qubit (other than the output qubit) called the postselection qubit such that, for any $x \in\{0,1\}^{n}$,

$-\operatorname{Pr}\left[\operatorname{post}_{n}(x)=0\right]>0$,

- if $x \in L, \operatorname{Pr}\left[C_{n}(x)=1 \mid \operatorname{post}_{n}(x)=0\right] \geq 2 / 3$,

- if $x \notin L, \operatorname{Pr}\left[C_{n}(x)=1 \mid \operatorname{post}_{n}(x)=0\right] \leq 1 / 3$,

where the event " $\operatorname{post}_{n}(x)=0$ " means that the classical outcome of the Z-measurement on the postselection qubit is 0 .

- $L \in$ PostBPP if there exists a polynomial-size randomized classical circuit $R_{n}$ with $n$ input bits that, for any $x \in\{0,1\}^{n}$, outputs $R_{n}(x)$, post ${ }_{n}(x) \in\{0,1\}$ such that

$-\operatorname{Pr}\left[\operatorname{post}_{n}(x)=0\right]>0$,

- if $x \in L, \operatorname{Pr}\left[R_{n}(x)=1 \mid \operatorname{post}_{n}(x)=0\right] \geq 2 / 3$,

- if $x \notin L, \operatorname{Pr}\left[R_{n}(x)=1 \mid \operatorname{post}_{n}(x)=0\right] \leq 1 / 3$.

We use the notation $\operatorname{post}_{n}(x)=0$ both in the quantum and classical settings, but the meaning will be clear from the context. Another important class is the polynomial hierarchy $\mathrm{PH}=\bigcup_{j \geq 1} \Delta_{j}^{p}$. Here, $\Delta_{1}^{p}=\mathrm{P}$ and $\Delta_{j+1}^{p}=\mathrm{P}^{\mathrm{N} \Delta_{j}^{p}}$ for any $j \geq 1$, where $\mathrm{P}$ is the class of languages decided by polynomial-time classical algorithms and $\mathrm{N} \Delta_{j}^{p}$ is the non-deterministic class associated to $\Delta_{j}^{p}$ [15, 3 ]. It is widely believed that $\mathrm{PH} \neq \Delta_{j}^{p}$ for any $j \geq 1$ [15]. As shown in [3], if PostBQP $\subseteq$ PostBPP, then $\mathrm{PH}=\Delta_{3}^{p}$, i.e., $\mathrm{PH}$ collapses to the third level. It can be shown that, in our setting of elementary gates and quantum circuits, this relationship also holds when the condition $\operatorname{Pr}\left[\operatorname{post}_{n}(x)=0\right]>0$ in the definition of PostBQP is replaced with the condition that, for some polynomial $q$ (depending only on $\left.C_{n}\right), \operatorname{Pr}\left[\operatorname{post}_{n}(x)=0\right] \geq 1 / 2^{q(n)}$. In the following, we adopt the latter condition.

\section{Commuting Quantum Circuits}

\subsection{Hardness of the Weak Simulation}

It is known that there exists a depth-3 quantum circuit with $n$ input qubits, $O(\operatorname{poly}(n))$ ancillary qubits, and $O(\operatorname{poly}(n))$ output qubits such that it is not weakly simulatable with respect to multiplicative error unless PH collapses to the third level [3]. We first analyze its weak simulatability with respect to additive error and show the following lemma: 
Lemma 1. There exists a depth-3 polynomial-size quantum circuit with $O(\operatorname{poly}(n))$ output qubits such that it is not weakly simulatable (with respect to additive error) unless $\mathrm{PH}$ collapses to the third level.

Proof. We assume that $\mathrm{PH}$ does not collapse to the third level. Then, as described above, PostBQP $\nsubseteq$ PostBPP. Let $L \in$ PostBQP $\backslash$ PostBPP. Then, there exists a polynomial-size quantum circuit $C_{n}$ with $n$ input qubits, $a=O(\operatorname{poly}(n))$ ancillary qubits, one output qubit, and one postselection qubit (and some polynomial $q$ ) such that, for any $x \in\{0,1\}^{n}$,

- $\operatorname{Pr}\left[\operatorname{post}_{n}(x)=0\right] \geq 1 / 2^{q(n)}$,

- if $x \in L, \operatorname{Pr}\left[C_{n}(x)=1 \mid \operatorname{post}_{n}(x)=0\right] \geq 2 / 3$,

- if $x \notin L, \operatorname{Pr}\left[C_{n}(x)=1 \mid \operatorname{post}_{n}(x)=0\right] \leq 1 / 3$.

As shown in [5], there exists a depth-3 polynomial-size quantum circuit $A_{n}$ with $n$ input qubits, $a+b$ ancillary qubits, and one output qubit such that, for any $x \in\{0,1\}^{n}$,

- if $x \in L, \operatorname{Pr}\left[A_{n}(x)=1 \mid \operatorname{qpost}_{n}(x)=0^{b+1}\right] \geq 2 / 3$,

- if $x \notin L, \operatorname{Pr}\left[A_{n}(x)=1 \mid \operatorname{qpost}_{n}(x)=0^{b+1}\right] \leq 1 / 3$,

where $b=O(\operatorname{poly}(n))$, the event "qpost $(x)=0^{b+1}$ " means that all classical outcomes of $Z$ measurements on the qubit corresponding to the postselection qubit of $C_{n}$ and particular $b$ qubits (other than the output qubit) are 0 . We call these $b+1$ qubits the postselection qubits of $A_{n}$. Since the probability of obtaining $0^{b}$ by $Z$-measurements on the $b$ qubits is $1 / 2^{b}$ [5], it holds that

$$
\operatorname{Pr}\left[\operatorname{qpost}_{n}(x)=0^{b+1}\right]=\frac{1}{2^{b}} \cdot \operatorname{Pr}\left[\operatorname{post}_{n}(x)=0\right] \geq \frac{1}{2^{b+q}} .
$$

We regard $A_{n}$, which has only one output qubit, as a new circuit with $b+2$ output qubits, where one of the output qubits is the original output qubit $q_{\text {out }}$ of $A_{n}$ and the others are the $b+1$ postselection qubits of $A_{n}$. We also denote this circuit as $A_{n}$. Thus, $A_{n}$ is a depth-3 polynomial-size quantum circuit with $O(\operatorname{poly}(n))$ output qubits. For any $x \in\{0,1\}^{n}$,

- $\operatorname{Pr}\left[A_{n}(x)=0^{b+1} 1\right]=\operatorname{Pr}\left[A_{n}(x)=1 \& \operatorname{zpost}_{n}(x)=0^{b+1}\right]$,

- $\operatorname{Pr}\left[A_{n}(x)=0^{b+1} 0\right]=\operatorname{Pr}\left[A_{n}(x)=0\right.$ \&qpost $\left._{n}(x)=0^{b+1}\right]$,

where, for simplicity, we assume that the last output qubit of $A_{n}$ is $q_{\text {out }}$. Thus, for any $x \in\{0,1\}^{n}$,

- if $x \in L, \operatorname{Pr}\left[A_{n}(x)=0^{b+1} 1\right] \geq 2 \cdot \operatorname{Pr}\left[\operatorname{qpost}_{n}(x)=0^{b+1}\right] / 3$,

- if $x \notin L, \operatorname{Pr}\left[A_{n}(x)=0^{b+1} 1\right] \leq \operatorname{Pr}\left[\operatorname{qpost}_{n}(x)=0^{b+1}\right] / 3$.

We can show that, if $A_{n}$ is weakly simulatable, then $L \in$ PostBPP. This contradicts the assumption that $L \notin$ PostBPP and completes the proof. The details can be found in Appendix A.1.

The proof method of Lemma 1 can be considered as an elaborated version of the one in [19]. As pointed out by Nishimura and Morimae [14], we note that their proof method in [11] based on the complexity class SBQP [9] can also be used to show the lemma.

The OR reduction circuit reduces the computation of the OR function on $b$ bits to that on $O(\log b)$ bits [7]: for any $b$-qubit input state $\left|x_{1}\right\rangle \cdots\left|x_{b}\right\rangle$ with $x_{j} \in\{0,1\}$, the circuit outputs $|0\rangle^{\otimes m}$ if $x_{j}=0$ for every $j$ and an $m$-qubit state orthogonal to $|0\rangle^{\otimes m}$ if $x_{j}=1$ for some $j$, where $m=\lceil\log (b+1)\rceil$. Besides the $b$ input qubits, the circuit has $m$ ancillary qubits as output qubits. The first part of the circuit is a layer consisting of $H$ gates on the ancillary qubits. The middle 


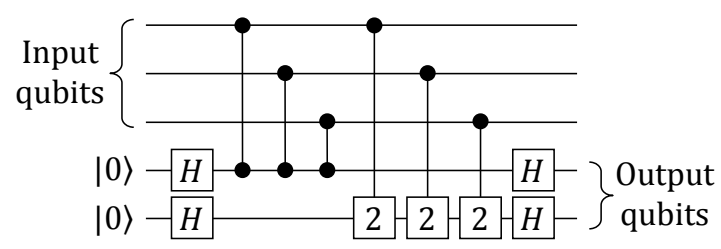

(a)

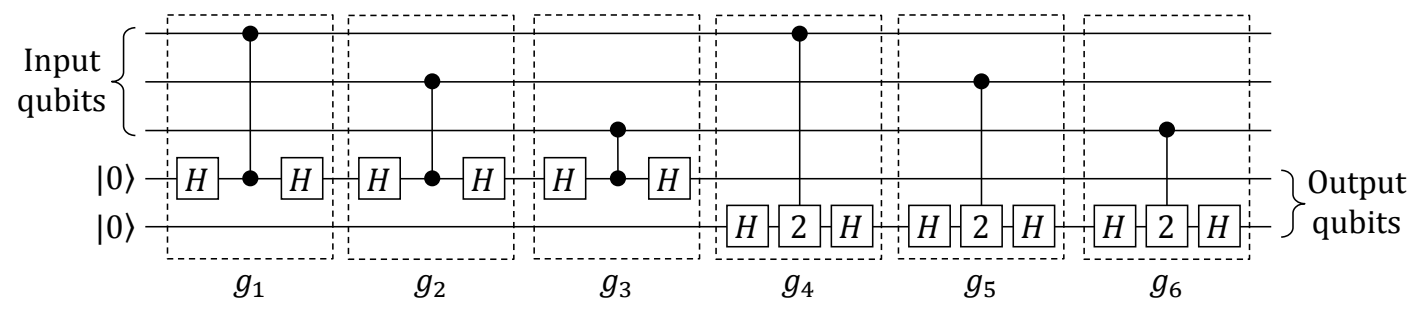

(b)

Figure 1: (a) The non-commuting OR reduction circuit, where $b=3$, the gate represented by two black circles connected by a vertical line is a $\Lambda Z$ gate, i.e., a controlled- $R\left(2 \pi / 2^{1}\right)$ gate, and the gate represented by " 2 " is an $R\left(2 \pi / 2^{2}\right)$ gate. (b) The commuting OR reduction circuit, where $b=3$.

part is a quantum circuit consisting of $b$ controlled- $R\left(2 \pi / 2^{k}\right)$ gates over all $1 \leq k \leq m$, where each gate uses an input qubit as the control qubit and an ancillary qubit as the target qubit. Such a gate is not an elementary gate, but it can be decomposed into a sequence of elementary gates. The last part is the same as the first one. We call the circuit the non-commuting OR reduction circuit. It is depicted in Fig. 1(a), where $b=3$.

An important observation is that the non-commuting OR reduction circuit can be transformed into a 2-local commuting quantum circuit. This is shown by considering a quantum circuit consisting of gates $g_{j}$ on two qubits, where each $g_{j}$ is a controlled- $R\left(2 \pi / 2^{k}\right)$ gate, which is in the non-commuting OR reduction circuit, sandwiched between Hadamard gates on the target qubit. Since $H H=I$ and controlled- $R\left(2 \pi / 2^{k}\right)$ gates are pairwise commuting gates on two qubits, the operation performed by the circuit is the same as that performed by the non-commuting OR reduction circuit and the gates $g_{j}$ are pairwise commuting gates on two qubits. We call the circuit the commuting OR reduction circuit. It is depicted in Fig. 1(b), where $b=3$. Combining this commuting OR reduction circuit with $A_{n}$ in the above proof implies the following lemma:

Lemma 2. There exists a commuting quantum circuit with $O(\log n)$ output qubits such that it is not weakly simulatable unless $\mathrm{PH}$ collapses to the third level.

Proof. As in the proof of Lemma 1, we can take $L \in$ PostBQP $\backslash$ PostBPP and obtain a depth-3 polynomial-size quantum circuit $A_{n}$ with $n$ input qubits, $a+b$ ancillary qubits, and $b+2$ output qubits such that, for any $x \in\{0,1\}^{n}$,

- if $x \in L, \operatorname{Pr}\left[A_{n}(x)=0^{b+1} 1\right] \geq 2 \cdot \operatorname{Pr}\left[\operatorname{qpost}_{n}(x)=0^{b+1}\right] / 3$,

- if $x \notin L, \operatorname{Pr}\left[A_{n}(x)=0^{b+1} 1\right] \leq \operatorname{Pr}\left[\operatorname{qpost}_{n}(x)=0^{b+1}\right] / 3$.

We construct a quantum circuit $E_{n}$ with $n$ input qubits, $a+b+m+1$ ancillary qubits, and $m+1$ output qubits as follows, where $m=\lceil\log (b+2)\rceil$. As an example, $E_{n}$ is depicted in Fig. 2(a), where $n=5, a=0$, and $b=2$ (and thus $m=2$ ).

1. Apply $A_{n}$ on $n$ input qubits and $a+b$ ancillary qubits, where the input qubits of $E_{n}$ are those of $A_{n}$. 


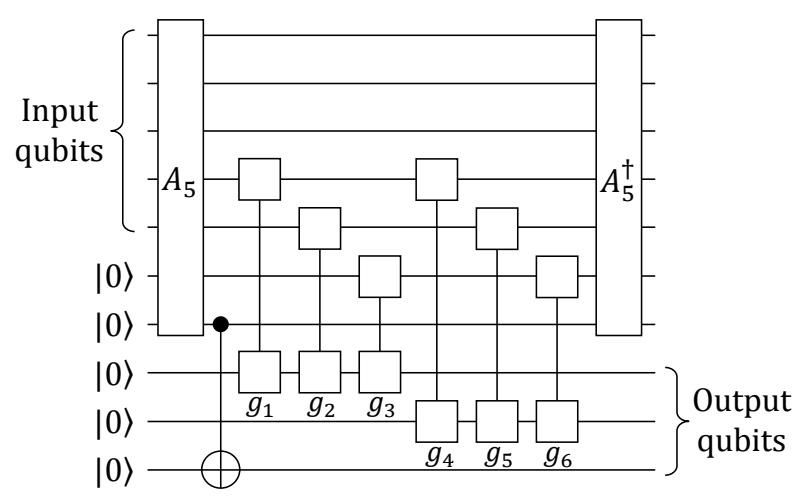

(a)

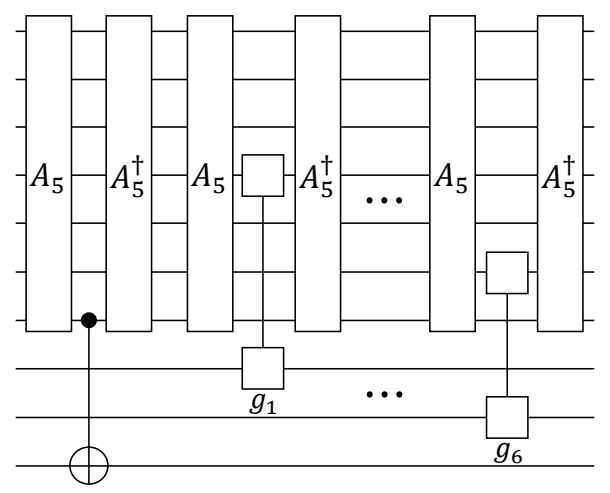

(b)

Figure 2: (a) Circuit $E_{n}$, where $n=5, a=0$, and $b=2$ (and thus $m=2$ ). The gate represented by a black circle and $\oplus$ connected by a vertical line is a $\Lambda X$ gate. The gates $g_{j}$ are the ones in Fig. 1. (b) The commuting quantum circuit based on $E_{n}$ in (a).

2. Apply a $\Lambda X$ gate on the last output qubit of $A_{n}$ and on an ancillary qubit (other than the ancillary qubits in Step 1), where the output qubit is the control qubit.

3. Apply the commuting OR reduction circuit on the other output qubits of $A_{n}$, i.e., the $b+1$ postselection qubits of $A_{n}$, and $m$ ancillary qubits (other than the ancillary qubits in Steps 1 and 2), where the postselection qubits are the input qubits of the OR reduction circuit.

4. Apply $A_{n}^{\dagger}$ as in Step 1.

The $m+1$ ancillary qubits used in Steps 2 and 3 are the output qubits of $E_{n}$. Step 4 does not affect the output probability distribution of $E_{n}$, but it allows us to construct the commuting quantum circuit described below. By the construction of $E_{n}$, for any $x \in\{0,1\}^{n}$,

$$
\operatorname{Pr}\left[A_{n}(x)=0^{b+1} 1\right]=\operatorname{Pr}\left[E_{n}(x)=0^{m} 1\right], \operatorname{Pr}\left[A_{n}(x)=0^{b+1} 0\right]=\operatorname{Pr}\left[E_{n}(x)=0^{m} 0\right] .
$$

This implies that $E_{n}$ is not weakly simulatable. The proof is the same as that of Lemma 1 except that the number of output qubits we need to consider is only $m+1=O(\log n)$.

We show that there exists a commuting quantum circuit with $m+1$ output qubits such that its output probability distribution is the same as that of $E_{n}$. We consider a quantum circuit consisting of gates $A_{n}^{\dagger} g A_{n}$ for any gate $g$ that is either a $\Lambda X$ gate in Step 2 of $E_{n}$ or $g_{j}$ in the commuting OR reduction circuit. The input qubits and output qubits of $E_{n}$ are naturally considered as the input qubits and output qubits of the new circuit, respectively. The circuit based on $E_{n}$ in Fig. 2(a) is depicted in Fig. 2(b). Since these gates $g$ in $E_{n}$ are pairwise commuting, so are the gates $A_{n}^{\dagger} g A_{n}$. Moreover, $A_{n}^{\dagger} g A_{n}$ acts on a constant number of qubits (in fact, on at most $2^{3}+1=9$ qubits) since the depth of $A_{n}$ is three, $g$ is on two qubits, and the number of qubits on which both $g$ and $A_{n}$ are applied is one. By the construction of the circuit, its output probability distribution is the same as that of $E_{n}$.

To complete the proof of Theorem 1, it suffices to show that the commuting quantum circuit in the proof of Lemma 2 is 5-local. To show this, we give the details of the depth-3 quantum circuit constructed by the method in [5]. The circuit is based on a one-qubit teleportation circuit. We adopt the teleportation circuit depicted in Fig. 3(a), which is obtained from the standard one by decomposing it into the elementary gates. If the classical outcomes of $Z$-measurements on the two qubits other than the output qubit are 0 , the output state is the same as the input state. We call 


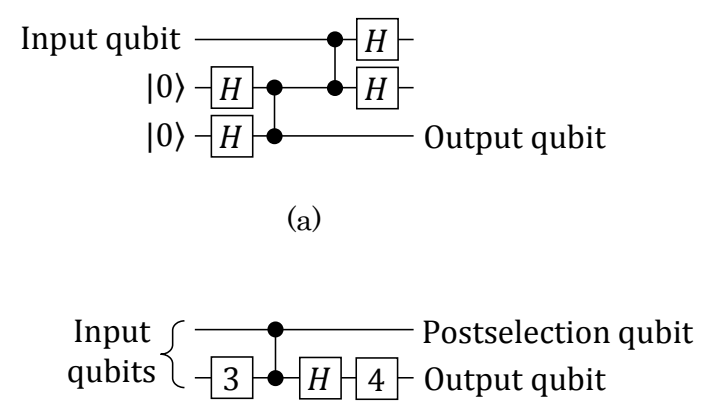

(b)
Layer 1 Layer 2 Layer 3

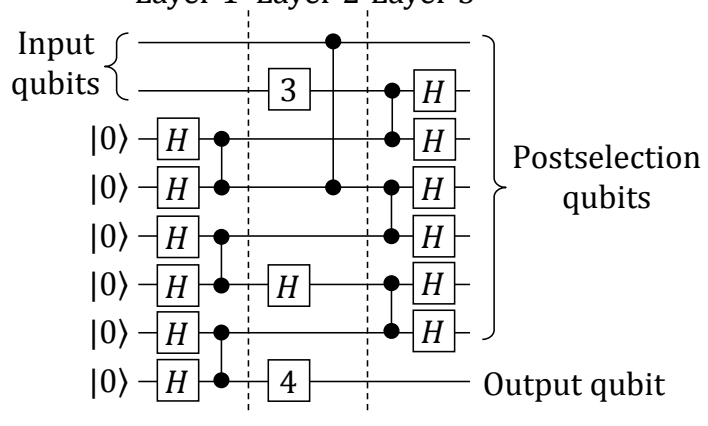

(c)

Figure 3: (a) The teleportation circuit. (b) An example of circuit $C_{n}$, where $n=2$ and $a=0$. The gate represented by $k \in \mathbb{N}$ is an $R\left(2 \pi / 2^{k}\right)$ gate. (c) Depth-3 circuit $A_{n}$ constructed from $C_{n}$ in (b) by the method in [5], where $b=6$ and thus the total number of postselection qubits is seven.

the first measured qubit, which is the input qubit, "the first teleportation qubit", and the second one "the second teleportation qubit".

For example, we consider the circuit depicted in Fig. 3(b) as $C_{n}$ in the proof of Lemma 1, where $n=2$ and $a=0$. The depth-3 circuit $A_{n}$ constructed from $C_{n}$ by the method in [5] is depicted in Fig. 3 (c), where $b=6$ and thus the total number of postselection qubits is seven. The first layer consists of the first halves of the teleportation circuits and the third consists of the last halves. The second layer consists of the gates in $C_{n}$. The teleportation qubits are the postselection qubits. If all classical outcomes of $Z$-measurements on the teleportation qubits are 0 , all teleportation circuits teleport their input states successfully and thus the output state is the same as that of $C_{n}$.

We will analyze $A_{n}^{\dagger} g A_{n}$ in the proof of Lemma 2, which implies the following lemma:

Lemma 3. For any gate $A_{n}^{\dagger} g A_{n}$ in the proof of Lemma 2, there exists a quantum circuit on at most five qubits that implements the gate.

Proof. We first consider the case when $g=g_{j}$ in the commuting OR reduction circuit. We divide this case into the following three cases, where we assume that $g$ is applied on a postselection qubit $q_{1}$ and an output qubit $q_{2}$ of $E_{n}$ :

- Case 1: $q_{1}$ is the first teleportation qubit (of a teleportation circuit).

- Case 2: $q_{1}$ is the second teleportation qubit (of a teleportation circuit).

- Case 3: $q_{1}$ is the postselection qubit corresponding to the one of $C_{n}$.

We obtain the desired circuit on at most five qubits by simplifying $A_{n}^{\dagger} g A_{n}$, where we represent $A_{n}$ as $L_{3} L_{2} L_{1}$, each of which is a layer of $A_{n}$. We consider Case 1 using an example of $A_{n}^{\dagger} g A_{n}$ depicted in Fig. 4(a), where $A_{n}$ is the circuit in Fig. 3(c), $g$ is a controlled- $R\left(2 \pi / 2^{k}\right)$ gate sandwiched between $H$ gates, and $q_{1}$ is the fourth qubit of $A_{n}$ from the top, which is the first teleportation qubit. By simplifying $L_{3}^{\dagger} g L_{3}$, we obtain the circuit depicted in Fig 4 (b). We can further simplify the circuit and obtain the desired circuit on five qubits $q_{1}, \ldots, q_{5}$ depicted in Fig. 4(c). In general, we can similarly simplify $A_{n}^{\dagger} g A_{n}$ and a similar analysis works for Cases 2 and 3 and the case when $g=\Lambda X$. The details can be found in Appendix A.2. 


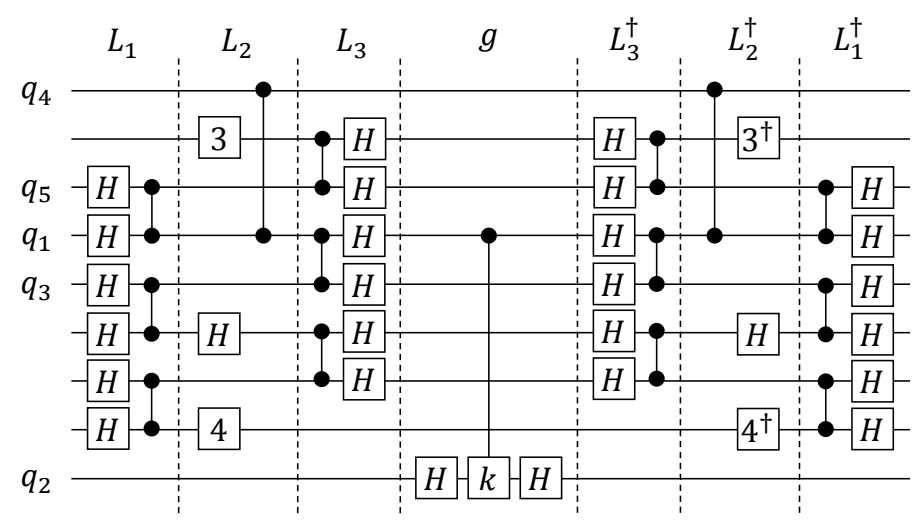

(a)

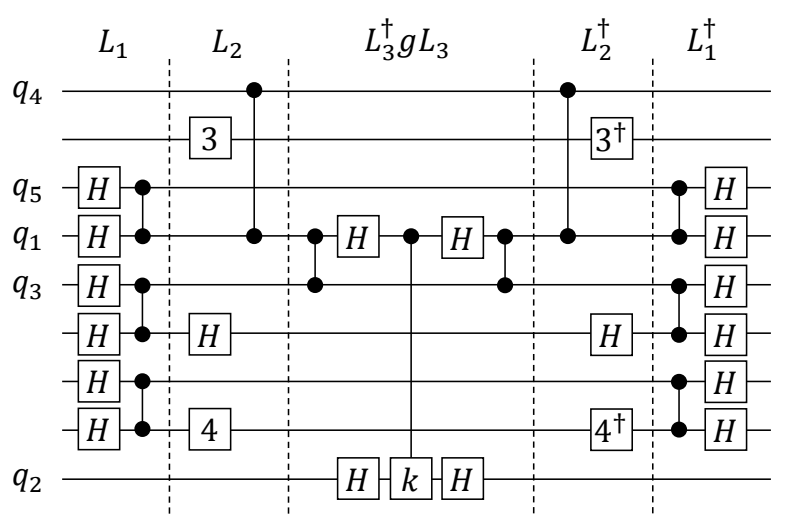

(b)

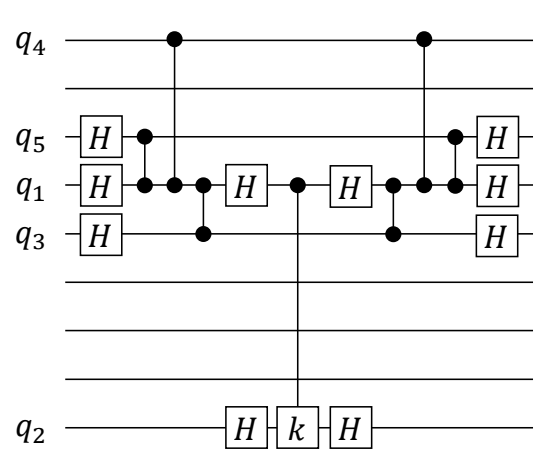

(c)

Figure 4: (a) Gate $A_{n}^{\dagger} g A_{n}$, where $A_{n}$ is the circuit in Fig. 3(c), $g$ is a controlled- $R\left(2 \pi / 2^{k}\right)$ gate sandwiched between $H$ gates, and $q_{1}$ is the fourth qubit of $A_{n}$ from the top. (b) The circuit obtained from $A_{n}^{\dagger} g A_{n}$ in (a) by simplifying $L_{3}^{\dagger} g L_{3}$. (c) The circuit on five qubits obtained from (b).

\subsection{Weak Simulatability of a Generalized Version}

The non-commuting OR reduction circuit with $b+1$ input qubits can be represented as $H^{\otimes m} D^{\prime} H^{\otimes m}$, where $m=\lceil\log (b+2)\rceil$ and $D^{\prime}$ is a quantum circuit consisting only of controlled- $R\left(2 \pi / 2^{k}\right)$ gates. Since $\Lambda X$ is $H \Lambda Z H$, we can represent the circuit in Theorem 1 as $\left(A_{n}^{\dagger} \otimes H^{\otimes(m+1)}\right) D^{\prime \prime}\left(A_{n} \otimes H^{\otimes(m+1)}\right)$, where $D^{\prime \prime}$ consists of $D^{\prime}$ and $\Lambda Z$, and $A_{n}$ is a depth-3 quantum circuit with $n$ input qubits, $a+b$ ancillary qubits, and $b+2$ output qubits. The output qubits of the whole circuit are the ancillary qubits on which $H^{\otimes(m+1)}$ is applied.

We generalize the circuit in Theorem 1. We assume that we are given two quantum circuits: $F_{n}$ is a quantum circuit with $n$ input qubits, $s=O(\operatorname{poly}(n))$ ancillary qubits, and $t(\leq n+s)$ output qubits and $D$ is a quantum circuit on $t+l$ qubits consisting of pairwise commuting gates that are diagonal in the $Z$-basis and act on a constant number of qubits, where $l=O(\log n)$. We consider the following quantum circuit, which can be represented as $\left(F_{n}^{\dagger} \otimes H^{\otimes l}\right) D\left(F_{n} \otimes H^{\otimes l}\right)$, with $n$ input qubits, $s+l$ ancillary qubits, and $l$ output qubits:

1. Apply $F_{n}$ on $n$ input qubits and $s$ ancillary qubits, where the input qubits of the whole circuit are those of $F_{n}$.

2. Apply $H^{\otimes l}$ on $l$ ancillary qubits (other than the ancillary qubits in Step 1). 
3. Apply $D$ on $t+l$ qubits, which are the output qubits of $F_{n}$ and the ancillary qubits in Step 2 .

4. Apply $H^{\otimes l}$ as in Step 2 and then apply $F_{n}^{\dagger}$ as in Step 1.

The output qubits are the ancillary qubits on which $H^{\otimes l}$ is applied. The circuit in Theorem 1 corresponds to the case when $F_{n}=A_{n}, D=D^{\prime \prime}, s=a+b, t=b+2$, and $l=m+1$.

When $F_{n}=H^{\otimes(n+s)}$ with arbitrary $s$ and $t,\left(F_{n}^{\dagger} \otimes H^{\otimes l}\right) D\left(F_{n} \otimes H^{\otimes l}\right)$ is weakly simulatable [3]. A simple generalization of the proof in [3] implies Theorem 2. In fact, we fix the state of the qubits other than the $O(\log n)$ output qubits on the basis of the assumption in Theorem 2 and then follow the change of the states of the output qubits. The details of the proof can be found in Appendix A.3. As described in Section 1, Theorem 2 implies an interesting suggestion on how to improve Theorem 1. Concretely speaking, a possible way to construct a 3- or 4-local commuting quantum circuit that is not weakly simulatable would be to somehow choose a depth-2 quantum circuit as $F_{n}$, but such a construction is impossible.

\section{Clifford Circuits}

As an application of the construction method for the circuit in Theorem 1, we consider Clifford circuits with $n$ input qubits, $O(\operatorname{poly}(n))$ ancillary qubits, and $O(\log n)$ output qubits. In this section, the ancillary qubits are allowed to be in a general product state (not restricted to a tensor product of $|0\rangle)$. As shown in [4, 8, such a Clifford circuit with only one output qubit is strongly simulatable. We first show that a simple extension of the proof in [4, 8] implies the strong simulatability of a Clifford circuit with $O(\log n)$ output qubits:

Lemma 4. Any Clifford circuit with $O(\operatorname{poly}(n))$ ancillary qubits in a general product state and with $O(\log n)$ output qubits is strongly simulatable.

The proof can be found in Appendix A.4.

In contrast to Lemma 4, it is known that there exists a Clifford circuit with $n$ input qubits, $O(\operatorname{poly}(n))$ ancillary qubits in a particular product state, and $O(\operatorname{poly}(n))$ output qubits such that it is not weakly simulatable with respect to multiplicative error unless $\mathrm{PH}$ collapses to the third level [8]. This is shown by using the fact that any PostBQP circuit can be simulated (in some sense) by a Clifford circuit. More precisely, let $L \in$ PostBQP and $C_{n}$ be a polynomial-size quantum circuit with $n$ input qubits, $a=O(\operatorname{poly}(n))$ ancillary qubits initialized to $|0\rangle$, one output qubit, and one postselection qubit (and some polynomial $q$ ) such that, for any $x \in\{0,1\}^{n}$,

- $\operatorname{Pr}\left[\operatorname{post}_{n}(x)=0\right] \geq 1 / 2^{q(n)}$,

- if $x \in L, \operatorname{Pr}\left[C_{n}(x)=1 \mid \operatorname{post}_{n}(x)=0\right] \geq 2 / 3$,

- if $x \notin L, \operatorname{Pr}\left[C_{n}(x)=1 \mid \operatorname{post}_{n}(x)=0\right] \leq 1 / 3$.

Then, there exists a Clifford circuit $A_{n}$ with $n$ input qubits, $a$ ancillary qubits initialized to $|0\rangle$, $b=O(\operatorname{poly}(n))$ ancillary qubits in a product state $|\varphi\rangle^{\otimes b}$, and one output qubit, where $|\varphi\rangle=$ $R(\pi / 4) H|0\rangle=\left(|0\rangle+e^{i \pi / 4}|1\rangle\right) / \sqrt{2}$, such that, for any $x \in\{0,1\}^{n}$,

- if $x \in L, \operatorname{Pr}\left[A_{n}(x)=1 \mid \operatorname{qpost}_{n}(x)=0^{b+1}\right] \geq 2 / 3$,

- if $x \notin L, \operatorname{Pr}\left[A_{n}(x)=1 \mid \operatorname{qpost}_{n}(x)=0^{b+1}\right] \leq 1 / 3$,

where the event "qpost ${ }_{n}(x)=0^{b+1}$ " means that all classical outcomes of $Z$-measurements on the qubit corresponding to the postselection qubit of $C_{n}$ and particular $b$ qubits (other than the output qubit) are 0 . We call these $b+1$ qubits the postselection qubits of $A_{n}$. We can 
show that $\operatorname{Pr}\left[\operatorname{qpost}_{n}(x)=0^{b+1}\right] \geq 1 / 2^{b+q}$. By using this property and $A_{n}$ obtained from $L \in$ PostBQP $\backslash$ PostBPP as in the proof of Lemma 1, we can show the following lemma, where the classical simulatability is defined with respect to additive error:

Lemma 5. There exists a Clifford circuit with $O(\operatorname{poly}(n))$ ancillary qubits in a particular product state and with $O(\operatorname{poly}(n))$ output qubits such that it is not weakly simulatable unless $\mathrm{PH}$ collapses to the third level.

As in the proof of Lemma 2, we construct a quantum circuit $E_{n}^{\prime}$ with $n$ input qubits and $a+b+m+1$ ancillary qubits by combining $A_{n}$ with the non-commuting OR reduction circuit as follows, where $m=\lceil\log (b+2)\rceil$ and the $m+1$ ancillary qubits are the output qubits of $E_{n}^{\prime}$. As an example, $E_{n}^{\prime}$ is depicted in Fig. 5(a), where $n=5, a=0$, and $b=2$.

1. Apply $A_{n}$ on $n$ input qubits, $a$ ancillary qubits initialized to $|0\rangle$, and $b$ ancillary qubits initialized to $|\varphi\rangle$, where the input qubits of $E_{n}^{\prime}$ are those of $A_{n}$.

2. Apply a $\Lambda X$ gate on the (original) output qubit of $A_{n}$ and an ancillary qubit (other than the ancillary qubits in Step 1), where the output qubit is the control qubit.

3. Apply the non-commuting OR reduction circuit on the $b+1$ postselection qubits of $A_{n}$ and $m$ ancillary qubits (other than the ancillary qubits in Steps 1 and 2), where the postselection qubits are the input qubits of the OR reduction circuit.

A direct application of the proof of Lemma 2 implies the following lemma:

Lemma 6. There exists a Clifford circuit combined with the OR reduction circuit as described above with $O(\operatorname{poly}(n))$ ancillary qubits in a particular product state and with $O(\log n)$ output qubits such that it is not weakly simulatable unless $\mathrm{PH}$ collapses to the third level.

We replace the non-commuting OR reduction circuit in Step 3 with a constant-depth OR reduction circuit with unbounded fan-out gates [7, where an unbounded fan-out gate can be considered as a sequence of CNOT gates with the same control qubit. It is easy to show that decomposing the unbounded fan-out gates into CNOT gates in the constant-depth OR reduction circuit yields a Clifford-1 circuit, which is a Clifford circuit augmented by a depth-1 non-Clifford layer. In particular, this procedure transforms the middle part of the non-commuting OR reduction circuit in Step 3, which is the only part that includes non-Clifford gates, into a quantum circuit that has CNOT gates and a depth-1 layer consisting of all gates in the middle part. The circuit obtained from the middle part in Fig. 5(a) is depicted in Fig. 5(b). This transformation with Lemma 6 implies Theorem 3 .

A similar argument implies that there exists a Clifford-2 circuit with $O(\operatorname{poly}(n))$ ancillary qubits in a particular product state and with only one output qubit such that it is not weakly simulatable unless PH collapses to the third level, where a Clifford-2 circuit has two depth-1 non-Clifford layers. Let $L \in$ PostBQP $\backslash$ PostBPP. We obtain $A_{n}$ as described above and combine it with a constantdepth quantum circuit for the OR function with unbounded fan-out gates [18]. By decomposing the unbounded fan-out gates into CNOT gates, the OR circuit can be transformed into a Clifford-2 circuit. Unfortunately, a combination of the circuits similar to the above construction has two output qubits. Thus, we construct two circuits with one output qubit. One circuit consists of $A_{n}$ and the OR circuit, where the input qubits of the OR circuit are the output qubit of $A_{n}$ and $b+1$ postselection qubits, and the output qubit of the OR circuit is the output qubit of the whole circuit. The other similarly consists of $X A_{n}$ and the OR circuit, where $X$ is applied on the output qubit of $A_{n}$. By a similar argument in [19], we can show that, if these two Clifford-2 circuits are weakly simulatable, then $L \in$ PostBPP. Thus, at least one of the circuits is not weakly simulatable. 


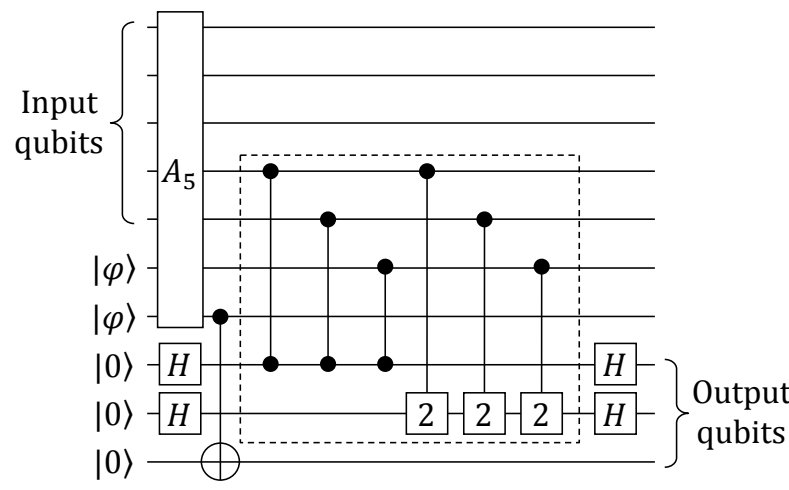

(a)

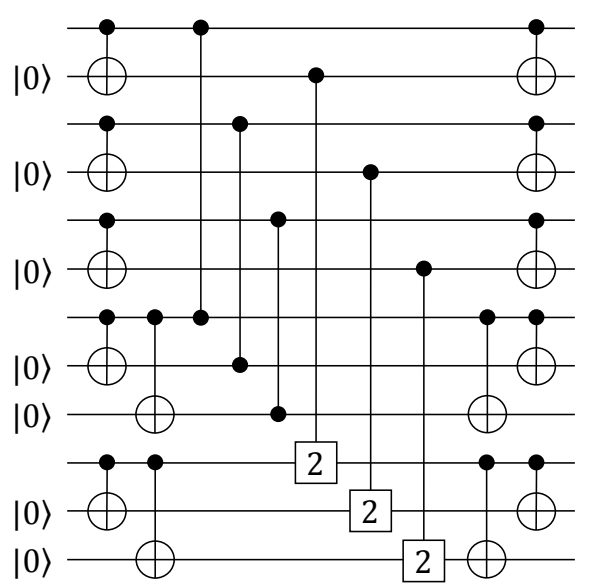

(b)

Figure 5: (a) Circuit $E_{n}^{\prime}$, where $n=5, a=0$, and $b=2$ (and thus $m=2$ ). The dashed box represents the middle part of the non-commuting OR reduction circuit. (b) The circuit that has CNOT gates and a depth-1 layer consisting of all gates in the middle part in (a). The qubits in state $|0\rangle$ are new ancillary qubits, which are not depicted in (a).

\section{Open Problems}

Interesting challenges would be to further investigate commuting quantum circuits and to consider closely related computational models. Some examples are as follows:

- Does there exist a 3- or 4-local commuting quantum circuit with $O(\log n)$ output qubits such that it is not weakly simulatable (under a plausible assumption)?

- Do the theorems in this paper hold when exponentially small error $1 / 2^{p(n)}$ is replaced with polynomially small error $1 / p(n)$ in the definitions of the classical simulatability?

- Can we apply the results on commuting quantum circuits to investigating the computational power of constant-depth quantum circuits?

\section{Acknowledgment}

We thank Harumichi Nishimura and Tomoyuki Morimae for pointing out to us the applicability of their proof method [11, which inspired us to realize that a slight modification of our proof method in the previous version of the present paper yields the stronger results described in this version.

\section{References}

[1] S. Aaronson. Quantum computing, postselection, and probabilistic polynomial-time. Proceedings of the Royal Society A, 461:3473-3482, 2005.

[2] S. Aaronson and A. Arkhipov. The computational complexity of linear optics. In Proceedings of the 43rd ACM Symposium on Theory of Computing (STOC), pages 333-342, 2011.

[3] M. J. Bremner, R. Jozsa, and D. J. Shepherd. Classical simulation of commuting quantum computations implies collapse of the polynomial hierarchy. Proceedings of the Royal Society A, 467:459-472, 2011. 
[4] S. Clark, R. Jozsa, and N. Linden. Generalized Clifford groups and simulation of associated quantum circuits. Quantum Information and Computation, 8(1\&2):106-126, 2008.

[5] S. Fenner, F. Green, S. Homer, and Y. Zhang. Bounds on the power of constant-depth quantum circuits. In Proceedings of Fundamentals of Computation Theory (FCT), volume 3623 of Lecture Notes in Computer Science, pages 44-55, 2005.

[6] Y. Han, L. A. Hemaspaandra, and T. Thierauf. Threshold computation and cryptographic security. SIAM Journal on Computing, 26(1):59-78, 1997.

[7] P. Høyer and R. Špalek. Quantum fan-out is powerful. Theory of Computing, 1(5):81-103, 2005.

[8] R. Jozsa and M. van den Nest. Classical simulation complexity of extended Clifford circuits. Quantum Information and Computation, 14(7\&8):633-648, 2014.

[9] G. Kuperberg. How hard is it to approximate the Jones polynomial?, 2009. arXiv:quant$\mathrm{ph} / 0908.0512$.

[10] I. L. Markov and Y. Shi. Simulating quantum computation by contracting tensor networks. SIAM Journal on Computing, 38(3):963-981, 2008.

[11] T. Morimae, H. Nishimura, K. Fujii, and S. Tamate. Classical simulation of DQC1 $1_{2}$ or DQC2 1 implies collapse of the polynomial hierarchy, 2014. arXiv:quant-ph/1409.6777.

[12] X. Ni and M. van den Nest. Commuting quantum circuits: efficient classical simulations versus hardness results. Quantum Information and Computation, 13(1\&2):54-72, 2013.

[13] M. A. Nielsen and I. L. Chuang. Quantum Computation and Quantum Information. Cambridge University Press, 2000.

[14] H. Nishimura and T. Morimae. Private communication, 2014.

[15] C. H. Papadimitriou. Computational Complexity. Addison Wesley, 1994.

[16] D. Shepherd. Binary matroids and quantum probability distributions, 2010. arXiv:quant$\mathrm{ph} / 1005.1744$.

[17] D. Shepherd and M. J. Bremner. Temporally unstructured quantum computation. Proceedings of the Royal Society A, 465:1413-1439, 2009.

[18] Y. Takahashi and S. Tani. Collapse of the hierarchy of constant-depth exact quantum circuits. In Proceedings of the 28th IEEE Conference on Computational Complexity (CCC), pages 168178, 2013.

[19] Y. Takahashi, T. Yamazaki, and K. Tanaka. Hardness of classically simulating quantum circuits with unbounded Toffoli and fan-out gates. Quantum Information and Computation, 14(13\&14):1149-1164, 2014.

[20] B. M. Terhal and D. P. DiVincenzo. Adaptive quantum computation, constant-depth quantum circuits and Arthur-Merlin games. Quantum Information and Computation, 4(2):134-145, 2004 .

[21] M. van den Nest. Classical simulation of quantum computation, the Gottesman-Knill theorem, and slightly beyond. Quantum Information and Computation, 10(3\&4):258-271, 2010.

[22] M. van den Nest. Simulating quantum computers with probabilistic methods. Quantum Information and Computation, 11(9\&10):784-812, 2011. 


\section{A Proofs}

\section{A.1 Proof of Lemma 1}

We assume that $A_{n}$ is weakly simulatable. Then, there exists a polynomial-size randomized classical circuit $R_{n}$ such that, for any $x \in\{0,1\}^{n}$ and $y \in\{0,1\}^{b+2}$,

$$
\left|\operatorname{Pr}\left[R_{n}(x)=y\right]-\operatorname{Pr}\left[A_{n}(x)=y\right]\right| \leq \frac{1}{2^{b+q+10}} .
$$

This implies that

$$
\begin{aligned}
& \operatorname{Pr}\left[A_{n}(x)=0^{b+1} 1\right]-\frac{1}{2^{b+q+10}} \leq \operatorname{Pr}\left[R_{n}(x)=0^{b+1} 1\right] \leq \operatorname{Pr}\left[A_{n}(x)=0^{b+1} 1\right]+\frac{1}{2^{b+q+10}}, \\
& \operatorname{Pr}\left[A_{n}(x)=0^{b+1} 0\right]-\frac{1}{2^{b+q+10}} \leq \operatorname{Pr}\left[R_{n}(x)=0^{b+1} 0\right] \leq \operatorname{Pr}\left[A_{n}(x)=0^{b+1} 0\right]+\frac{1}{2^{b+q+10}} .
\end{aligned}
$$

Since $\operatorname{Pr}\left[A_{n}(x)=0^{b+1} 1\right]+\operatorname{Pr}\left[A_{n}(x)=0^{b+1} 0\right]=\operatorname{Pr}\left[\right.$ qpost $\left._{n}(x)=0^{b+1}\right]$, it holds that

$$
\begin{aligned}
\left.\operatorname{Pr}_{\left[\operatorname{qpost}_{n}\right.}(x)=0^{b+1}\right]-\frac{1}{2^{b+q+9}} & \leq \operatorname{Pr}\left[R_{n}(x)=0^{b+1} 1\right]+\operatorname{Pr}\left[R_{n}(x)=0^{b+1} 0\right] \\
& \leq \operatorname{Pr}\left[\operatorname{qpost}_{n}(x)=0^{b+1}\right]+\frac{1}{2^{b+q+9}} .
\end{aligned}
$$

We construct a polynomial-size randomized classical circuit $S_{n}$ that implements the following classical algorithm with input $x \in\{0,1\}^{n}$ :

1. Compute $R_{n}(x)$.

2. (a) If $R_{n}(x)=0^{b+1} 1$, set $\operatorname{post}_{n}(x)=0$ and $S_{n}(x)=1$.

(b) If $R_{n}(x)=0^{b+1} 0$, set post ${ }_{n}(x)=0$ and $S_{n}(x)=0$.

(c) Otherwise, set $\operatorname{post}_{n}(x)=1$ and $S_{n}(x)=1$.

By the definition of $S_{n}$,

$$
\begin{aligned}
\operatorname{Pr}\left[\operatorname{post}_{n}(x)=0\right] & =\operatorname{Pr}\left[R_{n}(x)=0^{b+1} 1\right]+\operatorname{Pr}\left[R_{n}(x)=0^{b+1} 0\right] \\
& \geq \operatorname{Pr}\left[\operatorname{qpost}_{n}(x)=0^{b+1}\right]-\frac{1}{2^{b+q+9}} \\
& \geq \frac{1}{2^{b+q}}-\frac{1}{2^{b+q+9}}>0 .
\end{aligned}
$$

Moreover, for any $x \in\{0,1\}^{n}$,

$$
\operatorname{Pr}\left[S_{n}(x)=1 \mid \operatorname{post}_{n}(x)=0\right]=\frac{\operatorname{Pr}\left[R_{n}(x)=0^{b+1} 1\right]}{\operatorname{Pr}\left[R_{n}(x)=0^{b+1} 1\right]+\operatorname{Pr}\left[R_{n}(x)=0^{b+1} 0\right]} .
$$

If $x \in L$,

$$
\begin{aligned}
\operatorname{Pr}\left[S_{n}(x)=1 \mid \operatorname{post}_{n}(x)=0\right] & \geq \frac{\operatorname{Pr}\left[A_{n}(x)=0^{b+1} 1\right]-\frac{1}{2^{b+q+10}}}{\operatorname{Pr}\left[\operatorname{qpost}_{n}(x)=0^{b+1}\right]+\frac{1}{2^{b+q+9}}} \\
& \geq \frac{\frac{2}{3} \cdot \operatorname{Pr}\left[\operatorname{qpost}_{n}(x)=0^{b+1}\right]-\frac{1}{2^{b+q+10}}}{\operatorname{Pr}\left[\operatorname{qpost}_{n}(x)=0^{b+1}\right]+\frac{1}{2^{b+q+9}}} \\
& =\frac{2}{3}-\frac{7 \varepsilon}{3(1+2 \varepsilon)}>\frac{2}{3}-\frac{7}{3} \varepsilon>\frac{3}{5},
\end{aligned}
$$


where $\varepsilon=1 /\left(2^{b+q+10} \cdot \operatorname{Pr}\left[\operatorname{qpost}_{n}(x)=0^{b+1}\right]\right)$ and it holds that

$$
0<\varepsilon \leq \frac{1}{2^{b+q+10} \cdot \frac{1}{2^{b+q}}}=\frac{1}{2^{10}} .
$$

If $x \notin L$,

$$
\begin{aligned}
\operatorname{Pr}\left[S_{n}(x)=1 \mid \operatorname{post}_{n}(x)=0\right] & \leq \frac{\operatorname{Pr}\left[A_{n}(x)=0^{b+1} 1\right]+\frac{1}{2^{b+q+10}}}{\operatorname{Pr}\left[\operatorname{qpost}_{n}(x)=0^{b+1}\right]-\frac{1}{2^{b+q+9}}} \\
& \leq \frac{\frac{1}{3} \cdot \operatorname{Pr}\left[\operatorname{qpost}_{n}(x)=0^{b+1}\right]+\frac{1}{2^{b+q+10}}}{\operatorname{Pr}\left[\operatorname{qpost}_{n}(x)=0^{b+1}\right]-\frac{1}{2^{b+q+9}}} \\
& =\frac{1}{3}+\frac{5 \varepsilon}{3(1-2 \varepsilon)}<\frac{2}{5} .
\end{aligned}
$$

The constants $2 / 3$ and $1 / 3$ in the definition of PostBPP can be replaced with $1 / 2+\delta$ and $1 / 2-\delta$, respectively, for any constant $0<\delta<1 / 2[3]$. Thus, $L \in$ PostBPP.

\section{A.2 Proof of Lemma 3}

- Case 1: $q_{1}$ is the first teleportation qubit (of a teleportation circuit).

We note that $g$ is on the set of qubits $\left\{q_{1}, q_{2}\right\}$ and that there is no gate on $q_{2}$ in each layer. All $\Lambda Z$ gates other than the one on $q_{1}$ and qubit $q_{3}$ in layer 3 are cancelled out in $L_{3}^{\dagger} g L_{3}$. Only the $\Lambda Z$ gate, which is not cancelled out, increases the number of qubits involved with $\left\{q_{1}, q_{2}\right\}$ by one. Thus, $L_{3}^{\dagger} g L_{3}$ is on $\left\{q_{1}, q_{2}, q_{3}\right\}$. By the construction of the teleportation circuit, there is no gate on $q_{3}$ in layer 2. Only one $\Lambda Z$ gate on $q_{1}$ and qubit $q_{4}$ in layer 2 increases the number of qubits involved with $\left\{q_{1}, q_{2}, q_{3}\right\}$ by one. Thus, $L_{2}^{\dagger} L_{3}^{\dagger} g L_{3} L_{2}$ is on at most four qubits. If a $\Lambda Z$ gate is on $q_{3}$ or $q_{4}$ and on another qubit, it is cancelled out in $L_{1}^{\dagger} L_{2}^{\dagger} L_{3}^{\dagger} g L_{3} L_{2} L_{1}$. Only one $\Lambda Z$ gate on $q_{1}$ and qubit $q_{5}$ in layer 1 increases the number of qubits involved with $\left\{q_{1}, q_{2}, q_{3}, q_{4}\right\}$ by one. Thus, $L_{1}^{\dagger} L_{2}^{\dagger} L_{3}^{\dagger} g L_{3} L_{2} L_{1}$ is on at most five qubits.

- Case 2: $q_{1}$ is the second teleportation qubit (of a teleportation circuit).

As an example, $A_{n}^{\dagger} g A_{n}$ is depicted in Fig. 6(a), where $A_{n}$ is the circuit in Fig. 3(c), $g$ is a controlled- $R\left(2 \pi / 2^{k}\right)$ gate sandwiched between $H$ gates, and $q_{1}$ is the second qubit of $A_{n}$ from the bottom, which is the second teleportation qubit. As in Case 1, there is no gate on $q_{2}$ in each layer and $L_{3}^{\dagger} g L_{3}$ is on $\left\{q_{1}, q_{2}, q_{3}\right\}$. The circuit obtained from $A_{n}^{\dagger} g A_{n}$ in Fig. 6(a) by simplifying $L_{3}^{\dagger} g L_{3}$ is depicted in Fig. 6(b). By the construction of the teleportation circuit, there is no gate on $q_{1}$ in layer 2. If a $\Lambda Z$ gate is on $q_{3}$ and a qubit in layer 2 , it is cancelled out in $L_{2}^{\dagger} L_{3}^{\dagger} g L_{3} L_{2}$. Thus, gates in layer 2 do not increase the number of qubits involved with $\left\{q_{1}, q_{2}, q_{3}\right\}$. In layer 1 , a $\Lambda Z$ gate on $q_{1}$ and qubit $q_{4}$ increases the number of qubits involved with $\left\{q_{1}, q_{2}, q_{3}\right\}$ by one, and so does a $\Lambda Z$ gate on $q_{3}$ and qubit $q_{5}$. In particular, the latter happens only when an $H$ gate is on $q_{3}$ in layer 2. This is because, when any other gate, i.e., a $\Lambda Z$ or $R\left( \pm 2 \pi / 2^{k}\right)$ gate, is on $q_{3}$ in layer 2 , the gate is cancelled out in $L_{2}^{\dagger} L_{3}^{\dagger} g L_{3} L_{2}$ and thus a $\Lambda Z$ gate on $q_{3}$ and qubit $q_{5}$ is also cancelled out in $L_{1}^{\dagger} L_{2}^{\dagger} L_{3}^{\dagger} g L_{3} L_{2} L_{1}$. Thus, $L_{1}^{\dagger} L_{2}^{\dagger} L_{3}^{\dagger} g L_{3} L_{2} L_{1}$ is on at most five qubits. The circuit obtained from $A_{n}^{\dagger} g A_{n}$ in Fig. 6.(b) is depicted in Fig. 6. (c).

- Case 3: $q_{1}$ is the postselection qubit corresponding to the one of $C_{n}$.

Similar to the above cases, there is no gate on $q_{2}$ in each layer. By the construction of $A_{n}$, there is no gate on $q_{1}$ in layer 3 . Thus, it suffices to consider only $L_{2} L_{1}$. Since $g$ is on two qubits and the number of qubits on which both $g$ and $L_{2} L_{1}$ are applied is one, $L_{1}^{\dagger} L_{2}^{\dagger} g L_{2} L_{1}$ is on at most $2^{2}+1=5$ qubits. 


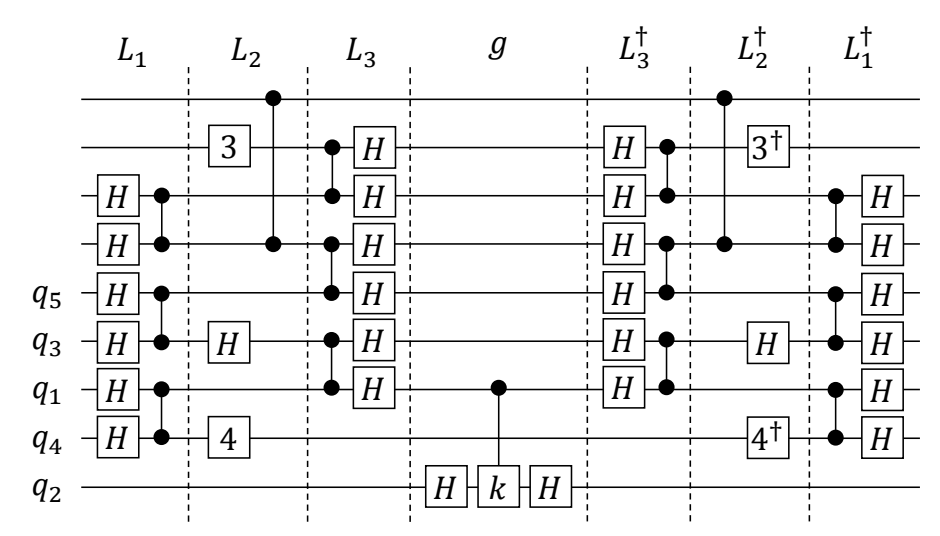

(a)

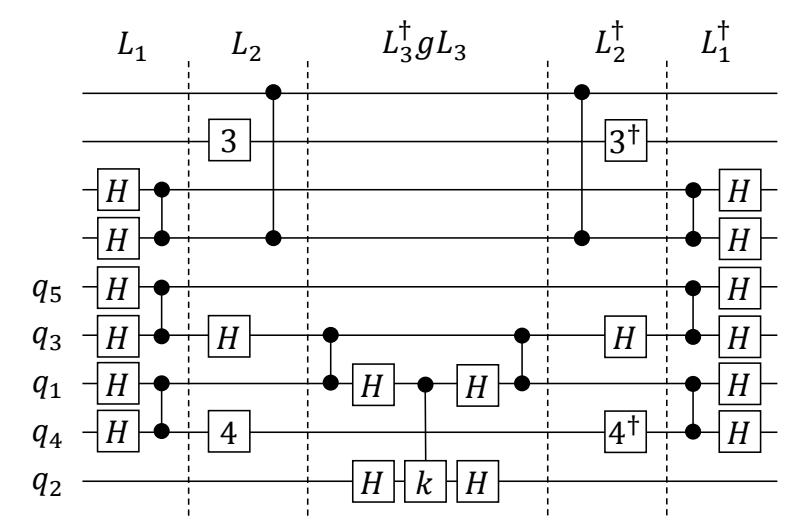

(b)

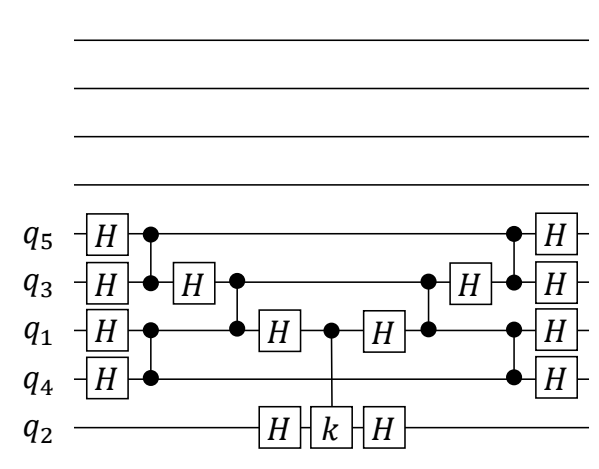

(c)

Figure 6: (a) Gate $A_{n}^{\dagger} g A_{n}$, where $A_{n}$ is the circuit in Fig. 3(c), $g$ is a controlled- $R\left(2 \pi / 2^{k}\right)$ gate sandwiched between $H$ gates, and $q_{1}$ is the second qubit of $A_{n}$ from the bottom. (b) The circuit obtained from $A_{n}^{\dagger} g A_{n}$ in (a) by simplifying $L_{3}^{\dagger} g L_{3}$. (c) The circuit on five qubits obtained from (b).

The analysis for Case 3 also works for the case when $g=\Lambda X$ in Step 2 of $E_{n}$.

\section{A.3 Proof of Theorem 2}

Let $|x\rangle$ be an $n$-qubit input state, where $x \in\{0,1\}^{n}$. Moreover, let

$$
F_{n}|x\rangle|0\rangle^{\otimes s}=\sum_{z \in\{0,1\}^{t}} \alpha_{x, z}|z\rangle\left|\psi_{x, z}\right\rangle
$$

where $\alpha_{x, z} \in \mathbb{C}$ and $\left|\psi_{x, z}\right\rangle$ is an $(n+s-t)$-qubit state. Then,

$$
\left(F_{n}^{\dagger} \otimes H^{\otimes l}\right) D\left(F_{n} \otimes H^{\otimes l}\right)|x\rangle|0\rangle^{\otimes(s+l)}=\frac{1}{\sqrt{2^{l}}}\left(F_{n}^{\dagger} \otimes H^{\otimes l}\right) \sum_{z \in\{0,1\}^{t}, w \in\{0,1\}^{l}} \alpha_{x, z} D|z\rangle\left|\psi_{x, z}\right\rangle|w\rangle .
$$

Since $D$ consists only of gates that are diagonal in the $Z$-basis, $D|z\rangle|w\rangle=e^{i f(z, w)}|z\rangle|w\rangle$ for some value $f(z, w)$ computed from the diagonal elements of $D$. Thus, the above state is

$$
\frac{1}{\sqrt{2^{l}}}\left(F_{n}^{\dagger} \otimes H^{\otimes l}\right) \sum_{z \in\{0,1\}^{t}, w \in\{0,1\}^{l}} \alpha_{x, z} e^{i f(z, w)}|z\rangle\left|\psi_{x, z}\right\rangle|w\rangle
$$


Thus, for any $y \in\{0,1\}^{l}$, the probability that $\left(F_{n}^{\dagger} \otimes H^{\otimes l}\right) D\left(F_{n} \otimes H^{\otimes l}\right)$ outputs $y$, which is represented as

is computed as

$$
\operatorname{Pr}\left[\left(F_{n}^{\dagger} \otimes H^{\otimes l}\right) D\left(F_{n} \otimes H^{\otimes l}\right)(x)=y\right],
$$

$$
\begin{aligned}
& \frac{1}{2^{l}} \sum_{z, z^{\prime} \in\{0,1\}^{t}, w, w^{\prime} \in\{0,1\}^{l}} \alpha_{x, z^{\prime}}^{\dagger} \alpha_{x, z} e^{-i f\left(z^{\prime}, w^{\prime}\right)+i f(z, w)}\left\langle z^{\prime} \mid z\right\rangle\left\langle\psi_{x, z^{\prime}} \mid \psi_{x, z}\right\rangle\left\langle w^{\prime}\left|H^{\otimes l}\right| y\right\rangle\left\langle y\left|H^{\otimes l}\right| w\right\rangle \\
& =\sum_{z \in\{0,1\}^{t}}\left|\alpha_{x, z}\right|^{2} \cdot \frac{1}{2^{l}} \sum_{w, w^{\prime} \in\{0,1\}^{l}} e^{-i f\left(z, w^{\prime}\right)+i f(z, w)}\left\langle w^{\prime}\left|H^{\otimes l}\right| y\right\rangle\left\langle y\left|H^{\otimes l}\right| w\right\rangle .
\end{aligned}
$$

Let $p(n)$ be an arbitrary polynomial. By the assumption, there exists a polynomial-size randomized classical circuit $R_{n}$ such that, for any $x \in\{0,1\}^{n}$ and $z \in\{0,1\}^{t}$,

$$
\left|\operatorname{Pr}\left[R_{n}(x)=z\right]-\operatorname{Pr}\left[F_{n}(x)=z\right]\right|=\left.\left|\operatorname{Pr}\left[R_{n}(x)=z\right]-\right| \alpha_{x, z}\right|^{2} \mid \leq \frac{1}{2^{p(n)+t}} .
$$

We consider a polynomial-size randomized classical circuit $T_{n}$ that implements the following classical algorithm for generating the probability distribution

$$
\left\{\left(y, \operatorname{Pr}\left[\left(F_{n}^{\dagger} \otimes H^{\otimes l}\right) D\left(F_{n} \otimes H^{\otimes l}\right)(x)=y\right]\right)\right\}_{y \in\{0,1\}^{l}},
$$

where the input is $x \in\{0,1\}^{n}$ :

1. Compute $z_{0}=R_{n}(x) \in\{0,1\}^{t}$.

2. Compute the probability that $Z$-measurements on the state

$$
\frac{1}{\sqrt{2^{l}}} \sum_{w \in\{0,1\}^{l}} e^{i f\left(z_{0}, w\right)} H^{\otimes l}|w\rangle
$$

output $y$ for any $y \in\{0,1\}^{l}$.

3. Output $y \in\{0,1\}^{l}$ according to the probability distribution computed in Step 2.

The probability in Step 2 is represented as

$$
\frac{1}{2^{l}} \sum_{w, w^{\prime} \in\{0,1\}^{l}} e^{-i f\left(z_{0}, w^{\prime}\right)+i f\left(z_{0}, w\right)}\left\langle w^{\prime}\left|H^{\otimes l}\right| y\right\rangle\left\langle y\left|H^{\otimes l}\right| w\right\rangle .
$$

We can compute $f\left(z_{0}, w\right)$ using a polynomial-size classical circuit since $D$ has polynomially many gates $g$ and it is easy to classically compute $\gamma_{g} \in \mathbb{C}$ such that $g\left|z_{0}\right\rangle|w\rangle=\gamma_{g}\left|z_{0}\right\rangle|w\rangle$ by using the classical description of $D$, which includes information about the complex numbers defining $g$ and the qubit numbers on which $g$ is applied. Moreover, since the state in Step 2 is only on $l=O(\log n)$ qubits, we can compute the probability in Step 2 up to an exponentially small additive error using a polynomial-size classical circuit. In the following, for simplicity, we assume that we can compute the probability exactly. Then, for any $y \in\{0,1\}^{l}$,

$$
\operatorname{Pr}\left[T_{n}(x)=y\right]=\sum_{z_{0} \in\{0,1\}^{t}} \operatorname{Pr}\left[R_{n}(x)=z_{0}\right] \cdot \frac{1}{2^{l}} \sum_{w, w^{\prime} \in\{0,1\}^{l}} e^{-i f\left(z_{0}, w^{\prime}\right)+i f\left(z_{0}, w\right)}\left\langle w^{\prime}\left|H^{\otimes l}\right| y\right\rangle\left\langle y\left|H^{\otimes l}\right| w\right\rangle .
$$

This implies that, for any $x \in\{0,1\}^{n}$ and $y \in\{0,1\}^{l}$,

$$
\begin{aligned}
\left|\operatorname{Pr}\left[T_{n}(x)=y\right]-\operatorname{Pr}\left[\left(F_{n}^{\dagger} \otimes H^{\otimes l}\right) D\left(F_{n} \otimes H^{\otimes l}\right)(x)=y\right]\right| & \leq\left.\sum_{z_{0} \in\{0,1\}^{t}}\left|\operatorname{Pr}\left[R_{n}(x)=z_{0}\right]-\right| \alpha_{x, z_{0}}\right|^{2} \mid \\
& \leq \frac{2^{t}}{2^{p(n)+t}}=\frac{1}{2^{p(n)}} .
\end{aligned}
$$

A similar argument works when we compute the probability in Step 2 up to an exponentially small additive error. Thus, $\left(F_{n}^{\dagger} \otimes H^{\otimes l}\right) D\left(F_{n} \otimes H^{\otimes l}\right)$ is weakly simulatable. 


\section{A.4 Proof of Lemma 4}

Let $C_{n}=G_{N} \cdots G_{1}$ be a Clifford circuit with $n$ input qubits, $a=O($ poly $(n))$ ancillary qubits, and $l=O(\log n)$ output qubits, where $N=O(\operatorname{poly}(n))$ and $G_{j}$ is $H, P$, or $\Lambda Z$. For any $x=x_{1} \cdots x_{n} \in$ $\{0,1\}^{n}$, let $\left|\psi_{x}\right\rangle=\left|x_{1}\right\rangle \cdots\left|x_{n}\right\rangle\left|\psi_{1}\right\rangle \cdots\left|\psi_{a}\right\rangle$ be an input state, where $\left|\psi_{j}\right\rangle$ is a one-qubit state. For any $y \in\{0,1\}^{l}$,

$$
\operatorname{Pr}\left[C_{n}(x)=y\right]=\left\langle\psi_{x}\left|C_{n}^{\dagger}\right| y\right\rangle\left\langle y\left|C_{n}\right| \psi_{x}\right\rangle=\left\langle\psi_{x}\left|C_{n}^{\dagger} X_{y}\right| 0\right\rangle^{\otimes l}\left\langle\left. 0\right|^{\otimes l} X_{y} C_{n} \mid \psi_{x}\right\rangle,
$$

where $X_{y}$ is the tensor product of $X$ and $I$ such that $X_{y}|0\rangle^{\otimes l}=|y\rangle$. As described in [12], it can be shown by induction on $l$ that

$$
|0\rangle^{\otimes l}\left\langle\left. 0\right|^{\otimes l}=\frac{1}{2^{l}} \sum_{S \subseteq\{1, \ldots, l\}} Z(S),\right.
$$

where $Z(S)$ is the tensor product of $Z$ and $I$ such that $Z$ is only on qubit $j \in S$. Thus,

$$
\operatorname{Pr}\left[C_{n}(x)=y\right]=\frac{1}{2^{l}} \sum_{S \subseteq\{1, \ldots, l\}}\left\langle\psi_{x}\left|G_{1}^{\dagger} \cdots G_{N}^{\dagger} X_{y} Z(S) X_{y} G_{N} \cdots G_{1}\right| \psi_{x}\right\rangle
$$

We can represent $G_{N}^{\dagger} X_{y} Z(S) X_{y} G_{N}$ as a tensor product of Pauli gates with some coefficient \pm 1 since $G_{N}$ is a Clifford gate and $X_{y} Z(S) X_{y}$ is a tensor product of Pauli gates (in fact, $Z$ and $I$ gates with some coefficient \pm 1 ). We repeat this transformation $N$ times and obtain

$$
\begin{aligned}
\operatorname{Pr}\left[C_{n}(x)=y\right] & =\frac{1}{2^{l}} \sum_{S \subseteq\{1, \ldots, l\}} \gamma^{S}\left\langle\psi_{x}\left|P_{1}^{S} \otimes \cdots \otimes P_{n+a}^{S}\right| \psi_{x}\right\rangle \\
& =\frac{1}{2^{l}} \sum_{S \subseteq\{1, \ldots, l\}} \gamma^{S}\left\langle x_{1}\left|P_{1}^{S}\right| x_{1}\right\rangle \cdots\left\langle x_{n}\left|P_{n}^{S}\right| x_{n}\right\rangle\left\langle\psi_{1}\left|P_{n+1}^{S}\right| \psi_{1}\right\rangle \cdots\left\langle\psi_{a}\left|P_{n+a}^{S}\right| \psi_{a}\right\rangle
\end{aligned}
$$

for some coefficient $\gamma^{S}$ and Pauli gates $P_{j}^{S}$. It is easy to construct a polynomial-time classical algorithm for obtaining $\gamma^{S}$ and $P_{j}^{S}$ for any $S \subseteq\{1, \ldots, l\}$. Moreover, since $l=O(\log n)$, it suffices to consider only polynomially many $S$. Thus, the above representation immediately implies a polynomial-time classical algorithm for computing $\operatorname{Pr}\left[C_{n}(x)=y\right]$. The marginal output probability can also be computed similarly and thus $C_{n}$ is strongly simulatable. 